\title{
Comparison of effects of corticotrophin and corticosteroids on pituitary-adrenal
} function

\author{
MARY E. CARTER AND V. H. T. JAMES \\ From St. Mary's Hospital and the Department of Chemical Pathology, St. Mary's Hospital Medical School, \\ London
}

It has long been accepted that pituitary-adrenal suppression occurs in the majority of patients treated with corticosteroids in doses of the order of $7.5 \mathrm{mg}$. or more daily of prednisolone or equivalent, although it is recognized that there is considerable patient variability. The mechanism of this process is not fully understood, but is attributed to cessation of ACTH release in response to the increased concentration of circulating glucocorticoid, and subsequent adrenal atrophy. Moreover, it is not at all clear why patients treated with long-term corticotrophin generally retain hypothalamic-pituitary adrenal responsiveness. We have shown (Carter and James 1970a) that patients receiving therapeutic daily doses of ACTH for periods of 2 years or more were able to produce a normal pituitary-adrenal response to the stress of insulin hypoglycaemia, and were also able to produce a normal increase in plasma cortisol levels while undergoing surgical operations without steroid cover (Carter and James, 1970b).

On the other hand, surprisingly, no reasonable therapeutic combination of ACTH with corticosteroids could be found which would preserve the integrity of the hypothalamic-pituitary-adrenal (HPA) axis in terms of stress responsiveness; in fact, the suppressive effect of steroids seems to be enhanced rather than offset, as had previously been supposed, by ACTH in the doses used (Carter and James, 1970c).

A series of studies has been made as part of a research programme with two main objectives:

(1) An attempt to find an improved therapeutic regime employing long-term corticotrophin and/or corticosteroids for patients suffering from chronically active rheumatic diseases-that is, one combining clinical efficacy as well as simplicity and acceptability of administration, with mitigation of side-effects, especially impairment of HPA function;
(2) A parallel aim to acquire from this work a better understanding of the different effects of these substances on the HPA axis.

This paper is concerned with the second objective, and deals with studies of the obvious features of corticotrophin therapy which it was thought might explain the different effects resulting from exogenous ACTH and steroid administration. In particular, we have investigated the dose, frequency, and time of administration, and the pattern of the plasma cortisol levels produced. We have also considered the role of two other steroids, androstenedione and dehydroepiandrosterone sulphate (d-sulphate), which are secreted by the adrenal cortex in response to ACTH, and the possibility of increased adrenocortical sensitivity to ACTH has also been studied.

\section{Material and methods}

Of 29 patients studied, eighteen were women and eleven men; 26 were suffering from classical or definite rheumatoid arthritis according to the American Rheumatism Association criteria (Ropes, Bennett, Cobb, Jacox, and Jessar, 1959) and three from psoriatic arthropathy. Their ages ranged from 28 to 70 years (mean 49). The mean duration from onset of disease was 9 years (range 1 to 27). They were not considered for corticosteroid or corticotrophin therapy until proper courses of treatment with salicylates and other antirheumatic drugs, as well as full supportive measures had failed to enable them to maintain an adequate functional status. They were then allocated to one of the following treatment groups for which they seemed best suited.

GROUP I

Corticotrophin given twice daily, morning and evening Five patients received their daily requirement of ACTH divided into two intramuscular injections.

GROUP II

Corticotrophin given once daily, each evening

Seven patients received their ACTH injection in the evenings, and these were compared with an earlier group 
of patients receiving their ACTH injection in the morning, our standard practice.

\section{GROUP III}

Delayed-absorption oral hydrocortisone in divided doses Five patients were treated with specially prepared tablets of hydrocortisone which produced a pattern of plasma cortisol which was exactly similar to that resulting from a single intramuscular injection of $\mathrm{ACTH} / \mathrm{cmc}$.

\section{GROUP IV}

Oral cortiscosteroids and dehydroepiandrosterone

Six patients were given their daily maintenance therapy of prednisolone 10 or $12.5 \mathrm{mg}$. divided, and were also given tablets of dehydroepiandrosterone sulphate $25 \mathrm{mg}$. daily.

\section{GROUP V}

\section{Oral corticosteroids and androstenedione}

Five male patients were treated with their normal daily requirement of prednisolone 10 or $12.5 \mathrm{mg}$. divided, and were also given a daily injection of androstenedione 25 mg.

All patients were started on these various forms of treatment as in-patients, so that they could be satisfactorily stabilized and accustomed to the first few insulin stress tests which were performed during this period; they subsequently came to the ward for half-day visits for follow-up assessment and insulin tests.

Since the metabolic effects of corticotrophin and corticosteroids were under comparison, it was important to establish equivalent dosages of the two drugs. While patients were in hospital, 24-hr urine collections were made throughout their stay in a metabolic ward where it could be reasonably certain that errors were minimal. Urine for the estimation of 17-oxogenic steroids was collected for periods of several consecutive days at a time from patients receiving varying doses of oral cortisol; the same procedure was applied to the same patients when they were receiving ACTH of various doses and types. Approximately equivalent dosage was then calculated on the basis of the daily excretion of 17-oxogenic steroids and on the comparative clinical efficacy of ACTH and cortisol.

Various types of corticotrophin were all given by the intramuscular route: 'Acthar Gel' (Armour), 'ACTH/cmc' (Crookes), 'Cortrophin ZN' (Organon), and 'Cortrosyn' (Organon).

The corticosteroids used were enteric-coated prednisolone, 'Delta Cortril' (Pfizer), hydrocortisone, and specially prepared hydrocortisone tablets which had delayed absorption (Glaxo). Tablets of dehydroepiandrosterone sulphate (Organon) and injections of androstenedione in arachis oil (Organon) were also used.

Plasma cortisol curves to determine the level and duration of activity of different types and doses of ACTH were performed over 48-hr periods, with blood sampling for cortisol levels every two hours.

Plasma cortisol was estimated by an automated fluorimetric technique (Townsend and James, 1968).
Urinary 17-oxogenic steroids were estimated by the method described by James and Caie (1964).

Insulin stress tests were performed as described by Greenwood, Landon, and Stamp (1966).

\section{Results}

The Table shows the mean daily urinary 17-oxogenic steroid excretion of twelve patients collected over a mean period of nine consecutive days per patient. These patients were receiving oral cortisol in doses of $25,40,50,60$, or $75 \mathrm{mg}$./day, and were compared with 26 patients receiving daily intramuscular injections of ACTH (Cortrophin ZN, Cortrosyn or Corticotrophin/cmc.), whose urine was collected over a mean period of seven consecutive days per patient. It can be seen that our commonly employed dose range of ACTH (15-40 units/day) is approximately equivalent in terms of 17-oxogenic excretion to cortisol 37.5 to $100 \mathrm{mg}$./day, that is to prednisolone 7.5 to $20 \mathrm{mg}$./day (see also Fig. 4).

Table Mean urinary 17-oxogenic steroid excretion (mg./24 hrs) from 38 patients receiving $A C T H$ or corticosteroids collected over a total of 282 days.

\begin{tabular}{|c|c|c|c|c|}
\hline \multicolumn{2}{|l|}{ Dosage } & \multicolumn{3}{|c|}{$\begin{array}{l}\text { Urinary 17-oxogenic steroids } \\
(\mathrm{mg} . / 24 \mathrm{hrs})\end{array}$} \\
\hline & & Mean & S.E. & $N$ \\
\hline ACTH (units day) & $\begin{array}{l}40 \\
30 \\
20 \\
15\end{array}$ & $\begin{array}{l}47 \\
41 \\
30 \\
21\end{array}$ & $\begin{array}{l}3 \cdot 9 \\
3 \cdot 3 \\
3 \cdot 4 \\
1 \cdot 0\end{array}$ & $\begin{array}{r}11 \\
8 \\
9 \\
7\end{array}$ \\
\hline $\begin{array}{l}\text { Oral cortisol } \\
\text { (prednisolone } \\
\quad \text { equivalents) } \\
\text { (mg./day) }\end{array}$ & $\begin{array}{l}75(15) \\
60(12) \\
50(10) \\
40(8) \\
30(6) \\
20(4)\end{array}$ & $\begin{array}{l}33 \\
24 \\
22 \\
18 \\
19 \\
15\end{array}$ & $\begin{array}{l}- \\
0.8 \\
1.7 \\
0.5 \\
0.6 \\
0.4\end{array}$ & $\begin{array}{r}3 \\
25 \\
8 \\
25 \\
30 \\
14\end{array}$ \\
\hline
\end{tabular}

FREQUENCY OF ADMINISTRATION OF ACTH

Fig. 1 shows that ACTH given in twice daily injections allows the patient to retain for the most part excellent pituitary-adrenal responsiveness to insulin hypoglycaemia. Some tests are slightly subnormal, but the overall results of the group are strictly comparable to those we obtained from patients given their daily requirement of ACTH in one morning injection (Carter and James, 1970a). It is difficult to explain the occasional test which seems to be at variance with the others (E.F. at 4 weeks, H.H. at 7 weeks). F.W. was unlike the other patients in showing transient suppression of response in the first tests; this pattern is similar to that shown by one other patient described elsewhere (Carter and James, 1970a). 

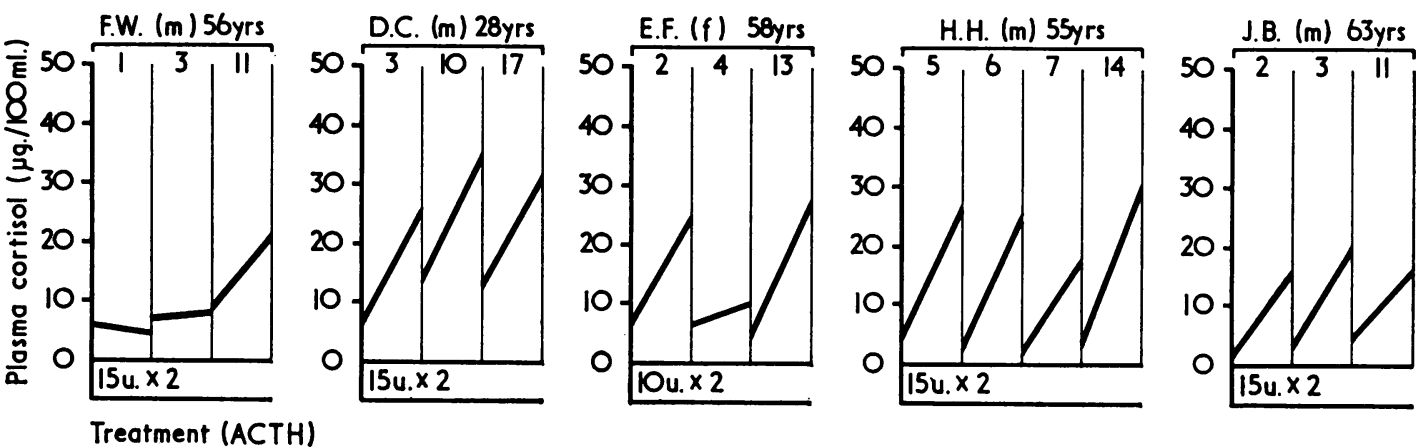

FIG. 1 Serial plasma cortisol responses to insulin hypoglycaemia in five patients receiving twice daily intramuscular injections of ACTH. Doses expressed in units/day at the foot of each graph.

Oblique lines show maximum increment in plasma cortisol for each test.

Tests carried out at intervals shown above graph in weeks.
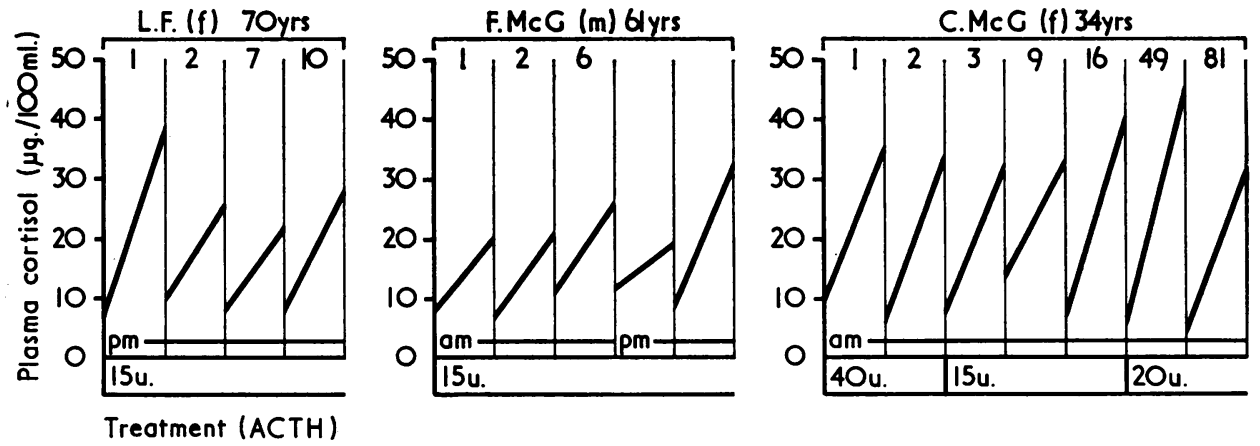

FIG. 2 Serial plasma cortisol responses to insulin hypoglycaemia in three patients receiving daily ntramuscular injections of $A C T H$. Doses expressed in units/day at the foot of each graph.

Oblique lines show maximum increment in plasma cortisol for each test.

Tests carried out at intervals shownabove graph in weeks. Morning or evening administration is indicated

TIME OF ADMINISTRATION OF ACTH

Fig. 2 contrasts insulin stress tests in patients given ACTH either in the morning or in the evening, and a third example is a patient who changed from morning to evening injections. Evening administration does not impair HPA function, responses being equally good whether ACTH is given in the morning or at night.

\section{COMPARISON OF CORTISOL CURVES \\ RESULTING FROM EXOGENOUS ACTH AND \\ CORTISOL}

When tablets of cortisol $(30 \mathrm{mg}$.) are taken orally there is a very steep rise in the blood cortisol level, which falls rapidly within 2 hours, and returns to the original level within 8 to 10 hours. The blood cortisol curve following an intramuscular injection of ACTH of comparable dose (15 units) is completely different, in that it rises more slowly and to half the level and, with some preparations, does not return to the original level before 26 hours. Two such curves are illustrated in Fig. 3 (overleaf).
With a view to obtaining blood cortisol curves with oral corticostercids similar to those resulting from an injecticn of ACTH, delayed-absorption tablets of hydrocortisone were specially prepared. Fig. 4 (overleaf) shows the closely matching curves obtained after an intramuscular injection of 15 units $\mathrm{ACTH} / \mathrm{cmc}$ and, separately, after the ingestion of these long-acting hydrocortisone tablets, $30 \mathrm{mg}$.

\section{'LONG-ACTING' ORAL HYDROCORTISONE}

Fig. 5 (overleaf) shows the results of serial insulin stress tests in four patients treated with these special delayed-absorption cortisol tablets in divided doses of $60 \mathrm{mg}$. daily for periods of 3 to 5 months. All stress responses are suppressed.

DEHYDROEPIANDROSTERONE SULPHATE OR ANDROSTENEDIONE WITH ORAL PREDNISOLONE Six patients receiving tablets of dehydroepiandrosterone sulphate $(25 \mathrm{mg}$.) in addition to prednisolone 


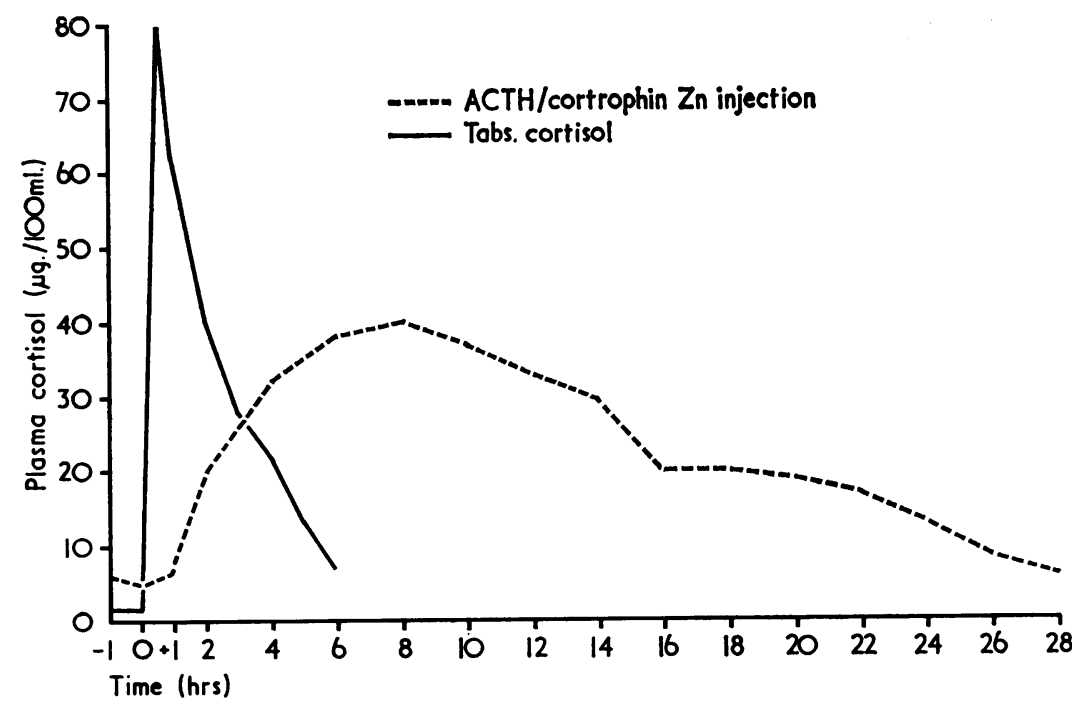

FIG. 3 Plasma cortisol curves after ingestion of oral cortisol $60 \mathrm{mg}$. at 11 a.m. (-), and separately in the same patient after an intramuscular injection at 10.30 a.m. of ACTH/Cortrophin $\mathrm{Zn} 30$ units (----).

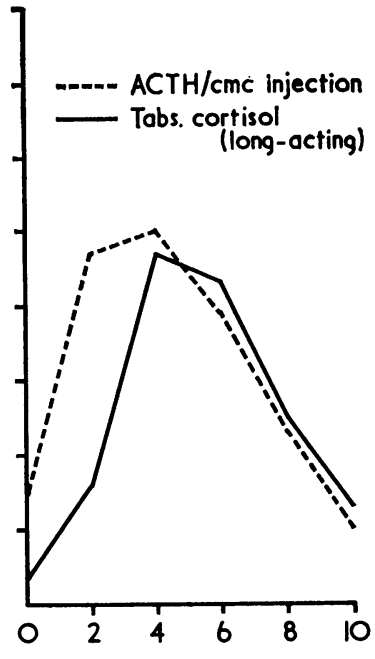

FIG. 4 Plasma cortisol curves after ingestion of oral 'longacting' cortisol $30 \mathrm{mg}$. (dexamethasone blocked) (-), and separately in the same patient after an intramuscular injection of $A C T H / c m c 15$ units (----).
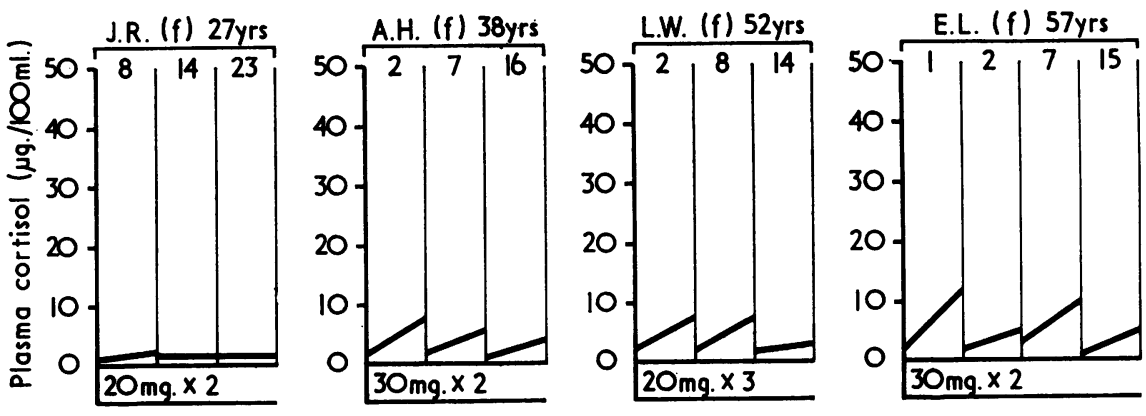

FIG. 5 Serial plasma cortisol responses to insulin hypoglycaemia in four female patients receiving oral 'long-acting' cortisol. Divided doses expressed as mg./day at foot of each graph. Oblique lines show maximum increment in plasma cortisol for each test. Tests carried out at intervals shown above graph in weeks.

Treatment ('long-acting' cortisol)

in divided doses of 10 or $12.5 \mathrm{mg}$. daily (Fig. 6, opposite) showed consistently flat responses to insulin hypoglycaemia, as did the five male patients who were given daily intramuscular injections of androstenedione $25 \mathrm{mg}$. with their daily prednisolone treatment (Fig. 7, opposite).

\section{ACUTE RESTORATION OF ADRENOCORTICAL FUNCTION}

Four patients who had been receiving prednisolone daily $(7 \cdot 5,10,12 \cdot 5$, and $17 \cdot 5 \mathrm{mg}$. respectively) were given 30 units ACTH twice daily for the 3 days before undergoing surgery. By examination of serial blood cortisol levels, it was confirmed that adrenocortical sensitivity to ACTH had been restored. On the 4th day, prednisolone $(20 \mathrm{mg}$.) was given and blood cortisol levels were estimated at regular intervals throughout surgery. The procedures carried out were pelvic floor repair, posterior tibialis tendon repair, Fowler's operation, and Hofmann's operation. No patient produced any alteration in blood cortisol level, implying that in these conditions ACTH release had not occurred.

\section{ADRENOCORTICAL SENSITIVITY TO ACTH}

Sixteen patients who had received continuous daily ACTH treatment in doses ranging from 20 to 40 units per day were studied in the way described by Landon, James, Wharton, and Friedman (1967) to assess threshold adrenocortical sensitivity. No indi- 

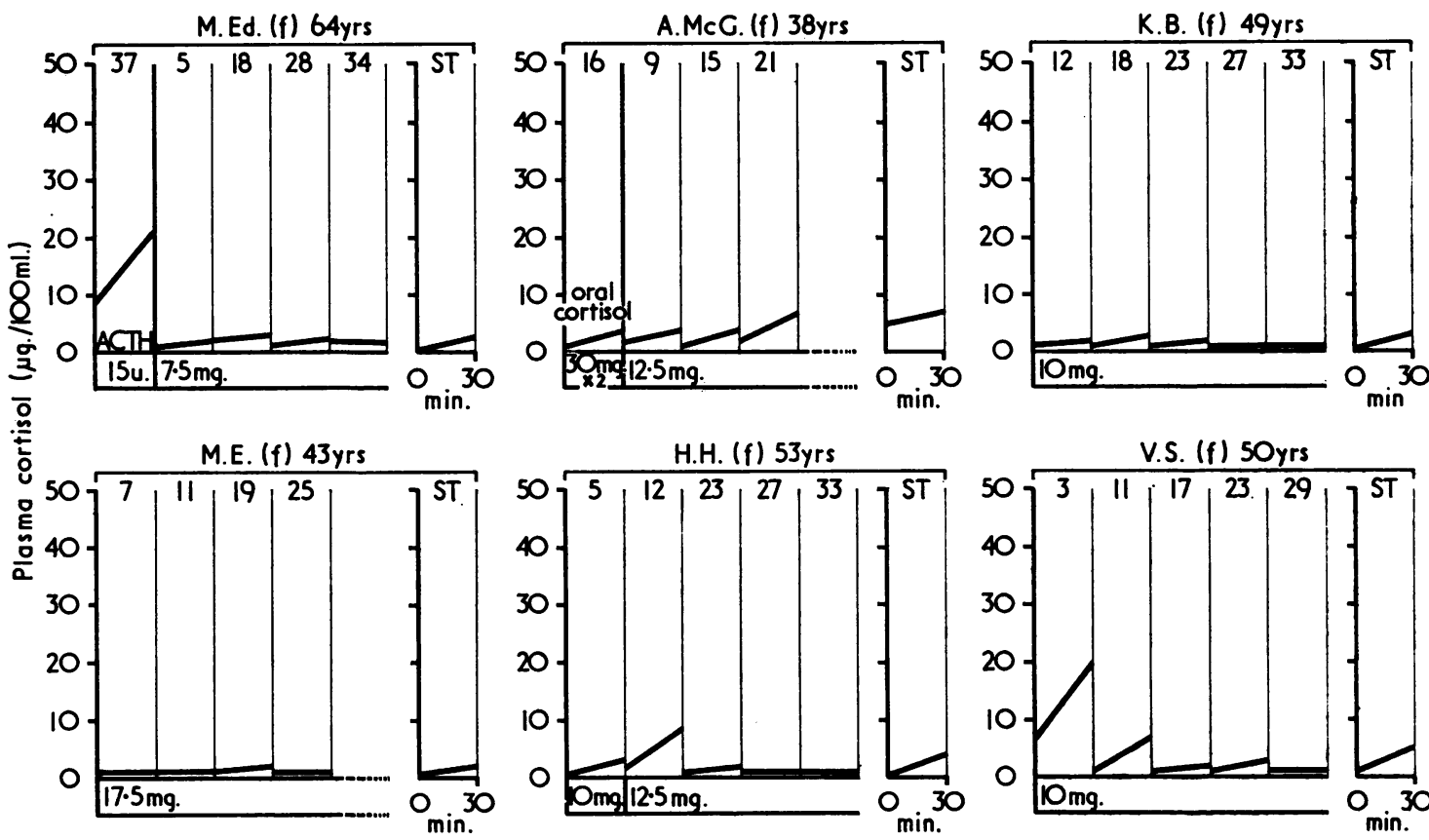

FIG. 6 Serial plasma cortisol responses to insulin hypoglycaemia in six female patients receiving oral prednisolone (divided doses expressed as mg./day at the foot of each graph), together with oral dehydroepiandrosterone (d-sulph) 25 mg. daily. Case M.Ed. has received ACTH 15 units daily for 37 weeks before the study. Case A. McG. had received oral cortisol $60 \mathrm{mg}$. daily for 16 weeks before the study. See left-hand columns. Right hand separate graph for each patient shows plasma cortisol response to intramuscular Synacthen $250 \mu \mathrm{g}$. at time of last insulin stress test.
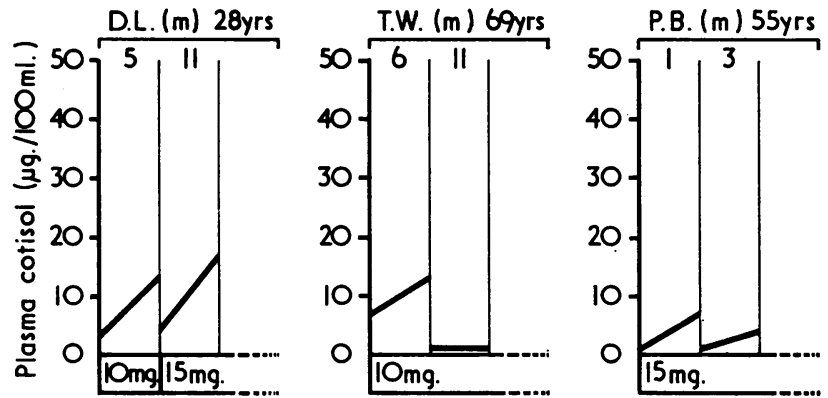

Treotment (prednisolone + androsțenedione)
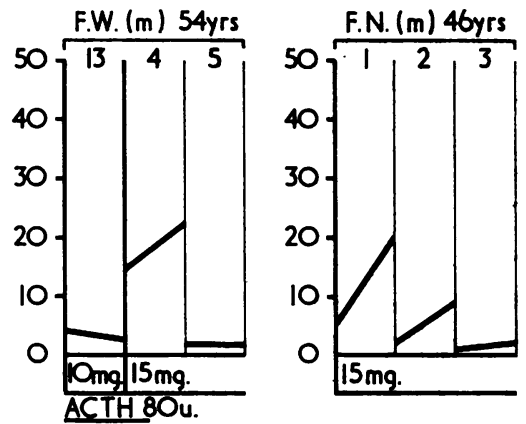

twice weekly

FIG. 7 Serial plasma cortisol responses to insulin hypoglycaemia in five male patients receiving oral prednisolone (divided doses expressed as $\mathrm{mg}$./day at the foot of each graph), together with intramuscular androstenedione $25 \mathrm{mg}$. daily.

Case F.W. had received treatment with prednisolone daily and ACTH twice weekly before study. See left-hand column.

cation of increased sensitivity to ACTH was obtained.

\section{Discussion}

None of the several factors investigated in this study appears to be of significance in the preservation of
HPA function in ACTH treated patients.

We have studied groups of patients receiving equivalent therapeutic doses of ACTH and of prednisolone and have concluded that the difference in HPA function in the two groups is not simply a question of the amount of steroid to which they are exposed. Neither does it seem likelv that the dif- 
ference is to be found in the frequency or time of administration of ACTH.

Bacon, Daly, Myles, and Savage (1968) have suggested that, when shorter acting ACTH preparations are given once daily, there is a period during the 24 hours in which the plasma cortisol levels are low, thus perhaps protecting the HPAaxis from suppression. However, twice daily administration of ACTH (Fig. 1) does not significantly impair HPA function. In addition, the time at which ACTH is given does not appear to be relevant, since even evening administration of ACTH does not cause HPA suppression (Fig. 2), even though this mode of therapy will tend to produce high plasma cortisol levels at a time when endogenous ACTH release occurs. In contrast, this is the time at which oral steroid will cause HPA suppression most effectively (Nichols, Nugent, and Tyler, 1965).

In seeking further differences between ACTH and corticosteroid therapy, we considered the possibility that the pattern of the plasma steroid might be of importance. As shown in Fig. 3, there is a marked difference between the pattern of blood cortisol after an intramuscular injection of ACTH and that after the ingestion of cortisol tablets, both the level reached and the duration of elevation being different. However, even when the plasma cortisol changes induced by ACTH were mimicked exactly by using delayed-absorption cortisol tablets, suppression occurred rapidly. The hypothesis that the pattern of plasma cortisol level is important in influencing the integrity of the HPA system therefore appears to be untenable.

Stimulation of the adrenal cortex with ACTH increases the secretion of steroids other than cortisol and $b c t$ th androstenedione and dehydroepiandrosterc ne s:Ilphate secretion is increased. It seemed worth considering the possibility that these steroids in some way modified the suppressive activity of endogenous cortisol on HPA function. To test this possibility, dehydroepiandrosterone sulphate $25 \mathrm{mg}$. daily or androstenedione $25 \mathrm{mg}$. daily was given to two groups of patients receiving divided doses of prednisolone 10 or $12.5 \mathrm{mg}$. daily. In both these groups, both the adrenal response to ACTH and the stress responses were severely impaired, and it was concluded that the adrenal androgens do not play a role in maintaining HPA responsiveness.

The status of the adrenal cortex in ACTH and corticosteroid-treated patients will of course be different. During ACTH treatment, the adrenal gland will remain responsive to endogenous and exogenous ACTH, and if larger doses of ACTH are given, will become hyperplastic. In contrast, as is well recognized, the adrenal gland in corticosteroidtreated patients is generally atrophic, and responds subnormally to administered ACTH.
It seemed possible, therefore, that the difference in stress responsiveness of ACTH and steroidtreated patients might find an explanation on this basis. Thus, if partial inhibition of ACTH release occurred with both types of therapy, the steroidtreated patients with some degree of adrenal atrophy would inevitably produce only a small or minimal increase in blood cortisol, whilst ACTH-treated patients, with healthy or possibly hyperplastic adrenals, would in contrast show good responses of blood cortisol. An attempt was made to test this hypothesis in our patients who had received steroids and who had been admitted to hospital for surgery. For 3 days immediately before the operation, ACTH was given daily to restore adrenal responsiveness. None of these patients showed any plasma steroid response to surgical stress. Neither could we detect any increase in threshold adrenal cortical sensitivity to ACTH in another group of patients who had been receiving continuous ACTH therapy. So far as we have been able to examine it, this explanation also appears to be untenable, although ideally, plasma ACTH levels should be determined in place of the indirect methods we have been obliged to employ.

In summary, then, we have been unable to find an explanation for the failure of ACTH to cause suppression of HPA function. Of the several factors studied, the dose, time of administration, role of other adrenal steroids, and adrenocortical sensitivity did not appear to be of major importance. Nevertheless, as we have shown elsewhere (Carter and James, 1970a), ACTH therapy does produce some abnormality of endogenous ACTH release, as revealed by the failure of some patients to respond when challenged with lysine vasopressin.

It seems likely that direct measurement of plasma ACTH levels may shed further light on these problems, the solution of which would have both theoretical and practical importance.

\section{Summary}

29 patients with chronic inflammatory polyarthritis receiving corticotrophin (ACTH) or corticosteroid therapy administered in varying schedules were studied, in order to seek an explanation for the different effects of these two drugs on the hypothalamic-pituitary adrenal system. The obvious possible reasons for the retention of pituitaryadrenal responsiveness in patients receiving longterm corticotrophin were investigated; results of these studies show that the dose of ACTH employed, the frequency of injections, the time of administration in relation to the diurnal rhythm of blood cortisol, and the cortisol pattern following an ACTH injection are apparently irrelevant. The other major adrenal hormones, dehydroepiandrosterone 
and androstenedione, which are released from the adrenal by ACTH, do not afford protection from suppression, at least in the doses used in this study.

We are pleased to acknowledge that this work was supported by grants from the Arthritis and Rheumatism Council for Research, and the Edgar Lawley Foundation, and we are also grateful to Armour, Crookes, Glaxo, and Organon Laboratories for additional assistance.

In particular we wish to thank Glaxo Laboratories for providing delayed-action cortisol tablets and the Organon Laboratories for compounding the dehydroepiandrosterone sulphate and androstenedione. Miss $\mathbf{K}$. M. Jones provided excellent technical assistance.

\section{References}

Bacon, P. A., Daly, J. R., Myles, A. B., And Savage, O. (1968) Ann. rheum. Dis., 27, 7 (Hypothalamo-pituitary adrenal function in patients on long-term adrenocorticotrophin therapy).

CARTER, M. E., AND JAMES, V. H. T. (1970a) Ibid., 29, 73 (Effect of corticotrophin therapy on pituitary-adrenal function).

corticotrophin treatment).

(1970c) Ann. rheum. Dis., 29, 409 (An attempt at combining corticotrophin with long-term corticosteroid therapy, with a view to preserving hypothalamic-pituitary-adrenal function).

Greenwood, F. C., LANDON, J., AND STAMP, T. C. B. (1966) J. clin. Invest., 45, 429 (The plasma sugar, free fatty acid, cortisol and growth hormone response to insulin: I. In control subjects).

JAMES, V. H. T., AND CAIE, E. (1964) J. clin. Endocr., 24, 180 (Determinations of urinary 17-hydroxycorticosteroids and their relation to cortisol secretion).

Landon, J., James, V. H. T., Wharton, M. J., AND Friedman, M. (1967) Lancet, 2, 697 (Threshold adrenocortical sensitivity in man and its possible application to corticotrophin bioassay).

Nichols, T., NUGENT, C. A., AND TYLER, F. H. (1965) J. clin. Endocr., 25, 343 (Diurnal variation in suppression of adrenal function by glucocorticoids).

Ropes, M. W., BenNetr, G. A., CoBb, S., JACOX, R., AND Jessar, R. A. (1959) Ann. rheum. Dis., 18, 49 (Diagnostic criteria for rheumatoid arthritis. 1958 revision).

TownSEND, J., AND JAMES, V. H. T. (1968) Steroids, 11, 497 (A semi-automated fluorimetric procedure for the determination of plasma corticosteroids). 